\title{
Long-path formation in a deformed microdisk laser
}

\author{
Susumu Shinohara, ${ }^{1}$ Takehiro Fukushima, ${ }^{2}$ Satoshi Sunada, ${ }^{3}$ Takahisa Harayama, ${ }^{4}$ and Kenichi Arai $^{1}$ \\ ${ }^{1}$ NTT Communication Science Laboratories, NTT Corporation, 2-4 Hikaridai, Seika-cho, Soraku-gun, Kyoto 619-0237, Japan \\ ${ }^{2}$ Department of Information and Communication Engineering, Okayama \\ Prefectural University, 111 Kuboki, Soja, Okayama 719-1197, Japan \\ ${ }^{3}$ Faculty of Mechanical Engineering, Institute of Science and Engineering, \\ Kanazawa University, Kakuma-machi, Kanazawa, Ishikawa 920-1192, Japan \\ ${ }^{4}$ Department of Applied Physics, School of Advanced Science and Engineering, \\ Waseda University, 3-4-1 Okubo, Shinjuku-ku, Tokyo 169-8555, Japan
}

(Dated: October 6, 2018)

\begin{abstract}
An asymmetric resonant cavity can be used to form a path that is much longer than the cavity size. We demonstrate this capability for a deformed microdisk equipped with two linear waveguides, by constructing a multiply reflected periodic orbit that is confined by total internal reflection within the deformed microdisk and outcoupled by the two linear waveguides. Resonant mode analysis reveals that the modes corresponding to the periodic orbit are characterized by high quality factors. From measured spectral and far-field data, we confirm that the fabricated devices can form a path about 9.3 times longer than the average diameter of the deformed microdisk.

PACS numbers: 42.55.Sa, 05.45.Mt, 42.55.Px, 42.60.Da
\end{abstract}

\section{INTRODUCTION}

In the past two decades, asymmetric resonant cavities (ARCs) have attracted considerable attention, because the introduction of asymmetry has proven useful in generating directional emissions, while maintaining high quality factors to some extent [1, 2]. From the viewpoint of dynamical billiard theory, the introduction of asymmetry generally accompanies the generation of raydynamical chaos [3] . Thorough theoretical and experimental investigations elucidated the relation between directional emission and ray-dynamical chaos [4 [6].

Although most of the previous work on ARCs has focused on directional emission, ARCs have another noteworthy capability, namely that of forming long optical paths by using multiple reflections at cavity interfaces. This capability was first demonstrated for a macroscopic (i.e., cm-sized) ARC with the aim of using it for gas sensing [ 7 -9], where long paths are important for increasing sensitivity. In that work, a three-dimensional ARC with diameter of $5.24 \mathrm{~cm}$ was fabricated of copper, and it was used to form a path of up to $6 \mathrm{~m}$. The idea of utilizing multiple reflections to form a long path can be found in relation to traditional gas sensing cavities consisting of two facing mirrors such as White cells and Herriott cells [10, 11].

We brought a similar idea to the micro-scale, and achieved a $2.79 \mathrm{~mm}$ path for a microcavity with an average diameter of $300 \mu \mathrm{m}$ [12], with the aim of using the path to realize a compact laser chaos device. Recently, laser chaos has attracted renewed interest, because of its usefulness as an entropy source for physical random number generation [13]. Laser chaos with a $\mathrm{GHz}$ bandwidth can be easily generated by a semiconductor laser with an external cavity for delayed optical feedback 14]. The external cavity must be at least a few $\mathrm{mm}$ long in order to obtain laser chaos suitable for random number generation. This requirement imposes a bottleneck on the device size, if we restrict ourselves to using a onedimensional external cavity. However, a two-dimensional external cavity with a sufficiently long path makes it possible to realize an ultra-small integrated laser chaos device whose footprint can be less than $1 \mathrm{~mm}^{2}$ [15].

Ref. [12] introduced a new type of cavity consisting of a deformed microdisk and two linear waveguides. This cavity enables the formation of a path much longer than the cavity diameter (see Fig. 11 (a) for the cavity shape). In contrast to a one-dimensional waveguide, long-path formation in two-dimensional slab cavity is highly nontrivial, because there is no transverse confinement, and only focusing at the cavity boundary prevents light from diffusing. In Ref. [12], the path formation was experimentally confirmed by injecting light from one of the linear waveguides and measuring the output from the other waveguide.

In this paper, we provide more detailed evidence for the path formation by performing systematic wave calculations for the cavity and carrying out experiments that allow direct theory-experiment comparisons. We also fabricate a relatively small device with an average diameter of $60 \mu \mathrm{m}$ (note that the average diameter of the device in Ref. 12 is $300 \mu \mathrm{m}$ ), whose experimental results can be directly compared with those of a resonant mode analysis. Firstly, we show that the resonant modes corresponding to the long path constitute a dominant group of high quality factor modes, implying that the formed path is well confined. By comparing results from the resonant mode analysis and those from Gaussian optical theory, we discuss the deviations from an ideal Gaussian beam caused by the presence of ray chaos. Secondly, we show that experimentally measured far-field patterns can be well reproduced by that of a resonant mode corresponding to the long path. Moreover, we reveal that measured modal spacings in optical spectra show excel- 

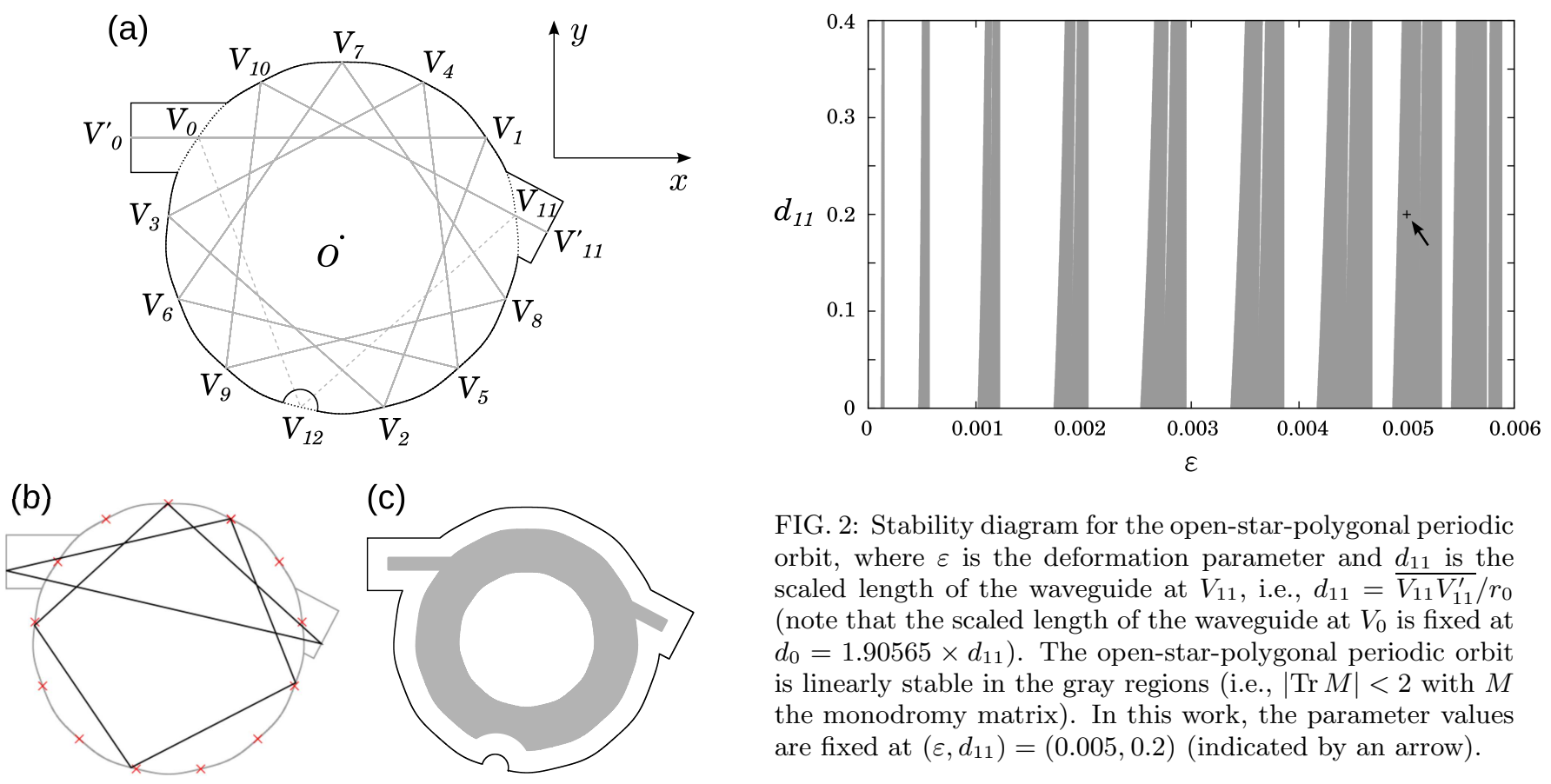

FIG. 2: Stability diagram for the open-star-polygonal periodic orbit, where $\varepsilon$ is the deformation parameter and $d_{11}$ is the scaled length of the waveguide at $V_{11}$, i.e., $d_{11}=\overline{V_{11} V_{11}^{\prime}} / r_{0}$ (note that the scaled length of the waveguide at $V_{0}$ is fixed at $\left.d_{0}=1.90565 \times d_{11}\right)$. The open-star-polygonal periodic orbit is linearly stable in the gray regions (i.e., $|\operatorname{Tr} M|<2$ with $M$ the monodromy matrix). In this work, the parameter values are fixed at $\left(\varepsilon, d_{11}\right)=(0.005,0.2)$ (indicated by an arrow).

FIG. 1: (Color online) (a) Deformed microdisk with two linear waveguides attached at $V_{0}$ and $V_{11}$ and a half-circular scatterer at $V_{12}$. (b) The stable periodic orbit that appears when the scatterer at $V_{12}$ is absent. (c) The contact area (gray region), where currents are injected.

lent agreement with the theoretical estimates assuming the long path. All these experimental results provide decisive evidence for the formation of a path that is about 9.3 times longer than the average diameter of a deformed microdisk. Additionally, we report that single-mode lasing occurs for continuous wave pumping.

This paper is organized as follows: In Sec. [I] we introduce our cavity design and present a stability analysis for a long periodic orbit. In Sec. III, we describe resonant mode analysis with particular attention to modes corresponding to a long periodic orbit. In Sec. IV] we provide experimental data for far-field and spectral measurements that confirm the existence of the designed long path. Sec. $\mathrm{\nabla}$ consists of a summary and discussion.

\section{CAVITY DESIGN}

We consider a cavity consisting of a deformed disk and two linear waveguides as shown in Fig. 1(a). The shape of the deformed disk is defined by

$$
r(\phi)=r_{0}[1-\varepsilon \sin (Q \phi)],
$$

where $(r, \phi)$ are the polar coordinates, and $r_{0}$ and $\varepsilon$ are the size and deformation parameters, respectively. In this paper, we fix $Q=13$, but the idea described below can apply to the other integer $Q$, provided that

the corresponding cavity has a star-polygonal periodic orbit. When we employ the shape given by Eq. (1) as the boundary for a dynamical billiard, we have a star-polygonal periodic orbit with 13 vertices labeled $V_{0}, V_{1}, \cdots, V_{12}$, as shown in Fig. 1 (a). These vertices correspond to the minimum curvature points of the boundary, and are defined by the polar angles $\phi_{m}=$ $\pi(17-4 m) / 26(m=0,1, \cdots, 12)$. As the star-polygonal orbit is traced until it closes, we have a $P=4$ times rotation around the origin $O$. In other words, the winding number of the star-polygonal orbit is $P / Q=4 / 13$. A linear stability analysis reveals that the periodic orbit is stable for $0 \leq \varepsilon \lesssim 0.00588$, and it is confined by total internal reflection when the refractive index of the cavity exceeds 1.76 (when we assume that the refractive index outside the cavity is 1 ).

At the vertex $V_{0}$ (resp. $V_{11}$ ), we attach a linear waveguide parallel to a periodic orbit segment $V_{0} V_{1}$ (resp. $\left.V_{10} V_{11}\right)$. This eliminates the star-polygonal periodic orbit. Instead, we have a self-retracing periodic orbit connecting both waveguide ends $V_{0}^{\prime}$ and $V_{11}^{\prime}$. This orbit includes the entire star-polygonal orbit except for the two line segments $\overline{V_{0} V_{12}}$ and $\overline{V_{11} V_{12}}$. It is this open-starpolygonal periodic orbit that we focus on in this paper. To prevent the other periodic orbits from appearing, we place a half-circular scatterer at the vertex $V_{12}$, as shown in Fig. 1(a). Without this scatterer, there is a stable periodic orbit as shown in Fig. 1 (b). The (one-way) path length $L_{*} r_{0}$ of the open-star-polygonal periodic orbit is given by

$$
L_{*} r_{0}=\left[2 N(1-\varepsilon) \sin \left(\pi \frac{P}{Q}\right)+d_{0}+d_{N}\right] r_{0},
$$

where $N$ is the index for the vertex with the waveguide (we assume that the other waveguide is attached to the 
vertex $\left.V_{0}\right)$. For the open-star-polygonal orbit shown in Fig. 11 (a), we have $L_{*} r_{0}=18.596 \times r_{0}$, that is, the path is about 9.3 times longer than the average cavity diameter.

The linear stability of the open-star-polygonal periodic orbit depends on three parameters, namely the deformation parameter $\varepsilon$ and the lengths of the two linear waveguides. For the waveguide attached to $V_{0}$ (resp. $\left.V_{11}\right)$, we use $d_{0}$ (resp. $\left.d_{11}\right)$ to denote the length scaled by $r_{0}$, i.e., $d_{i}=\overline{V_{i} V_{i}^{\prime}} / r_{0}(i=0,11)$. In what follows, we fix the ratio $d_{0} / d_{11}=1.90565$, and consider $d_{11}$ to be a free parameter, in addition to $\varepsilon$. The asymmetry of the waveguide lengths breaks the otherwise existing mirror symmetry of the cavity (this results in asymmetric emission patterns as seen in Figs. 4, 5] and 6).

We evaluated the monodromy matrix $M$ for the openstar-polygonal periodic orbit numerically for each set of $\varepsilon$ and $d_{11}$, and obtained the stability diagram shown in Fig. 2. where the periodic orbit is stable (i.e., $|\operatorname{Tr}(M)|<2$ ) in the gray regions. We hereafter fix the parameter values at $\left(\varepsilon, d_{11}\right)=(0.005,0.2)$ as indicated by the arrow in Fig. 2. We also fix the waveguide widths at $W_{0}=W_{11}=$ $0.395875 \times r_{0}$, so that they are much larger than the beam spot sizes at the waveguide ends.

\section{RESONANT MODE ANALYSIS}

We performed a numerical analysis of the resonant modes for the cavity defined in Fig. 11 (a) by using the boundary element method (BEM) [16]. The resonant modes are the solutions of the Helmholtz equa-

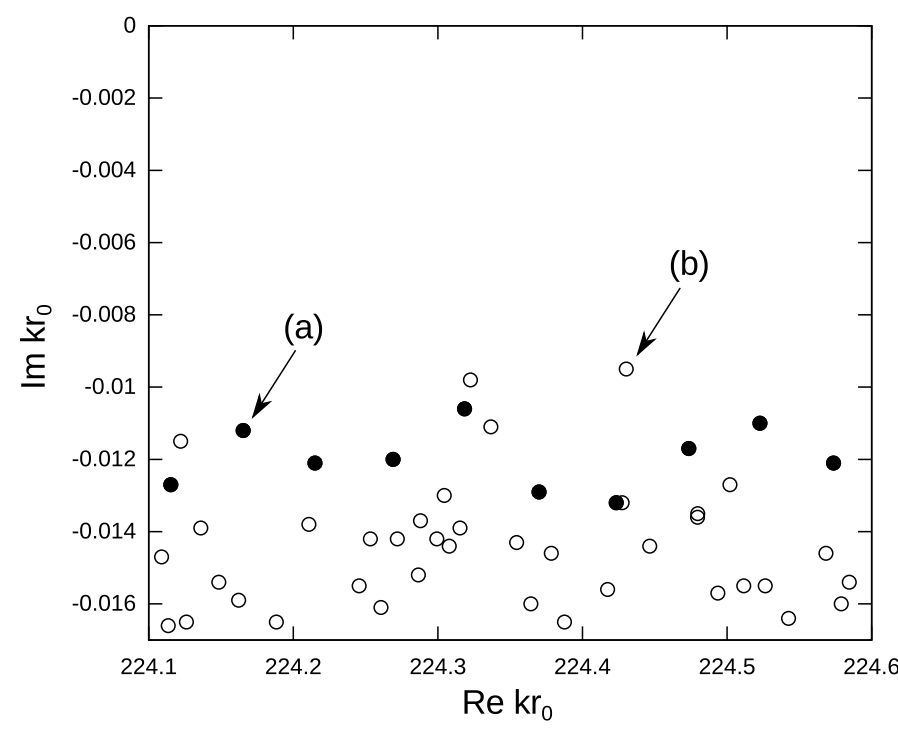

FIG. 3: The distribution of the resonances, where the star modes are plotted with filled circles $(\bullet)$. The star modes appear regularly with the average mode spacing $\Delta k r_{0}=0.0512$. The wave functions corresponding to (a) and (b) are shown in Figs. 4 (a) and 4 (b), respectively. tion $\left[\nabla^{2}+n(x, y)^{2} k^{2}\right] \psi(x, y)=0$, where $k$ is the wavenumber and $n(x, y)$ is the effective refractive index. We set $n(x, y) \equiv 3.3$ (GaAs) inside the cavity, while $n(x, y) \equiv 1$ (air) outside the cavity. As described later, we fabricated the cavity with an unstrained GaAs single-quantum-well structure. Since the emissions from these lasers are usually TE-polarized, we employ the cavity interface conditions for TE-polarization (i.e., both $\psi$ and $\left(1 / n^{2}\right) \partial \psi / \partial n$ are continuous at the cavity interface, where $\partial / \partial n$ is the derivative normal to the boundary). For the TE-polarization, the wave function $\psi$ represents the $z$-component of the magnetic field vector, i.e., $H_{z}=\operatorname{Re}\left[\psi e^{-i c k t}\right]$. At infinity, we assume the outgoing wave condition $\psi \propto e^{i k r} / \sqrt{r}$, which yields complex wavenumbers $k$ with $\operatorname{Im} k<0$.

Figure 3 shows the distribution of (dimensionless) complex wavenumbers $k r_{0}$ for resonant modes found numerically around $\operatorname{Re} k r_{0}=224.4$. This $\operatorname{Re} k r_{0}$ value corresponds to the cavity size $r_{0}=30 \mu \mathrm{m}$ when the wavelength is $\lambda=0.84 \mu \mathrm{m}$, which is one of our experimental conditions as discussed later.

According to Gaussian optical theory [17], Gaussian beam modes that are localized along the open-starpolygonal orbit are expected to exist when $\operatorname{Re} k r_{0}$ values are sufficiently large. We call these modes star modes, and we detected many of them in the BEM calculation (the star modes were clearly identified by their strong lo-
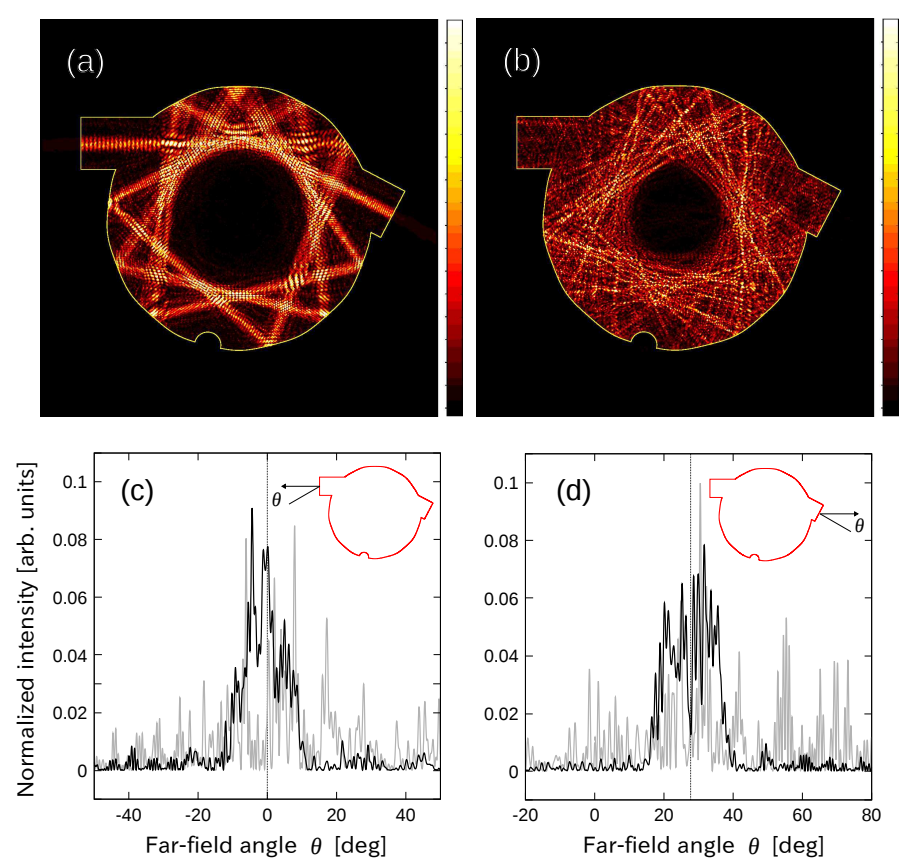

FIG. 4: (Color online) (a) and (b) show the intensity distributions of the wave functions for the resonances: (a) $k r_{0}=$ $224.165-i 0.011$ and (b) $k r_{0}=224.430-i 0.010$, where a lighter color indicates a higher intensity. (c) and (d), respectively, show far-field emission patterns for the mode shown in Fig. 4 (a) (black curves) and for the mode shown in Fig. 4 (b) (gray curves). 
calization along the open-star-polygonal orbit). In Fig. 3. the star modes are indicated by filled circles. We can see that the star modes constitute a dominant group among the high quality factor modes. For the star mode labeled (a) in Fig. 3 (i.e., $k r_{0}=224.165-i$ 0.011), we show the corresponding spatial intensity pattern of the wave function, $|\psi(x, y)|^{2}$, in Fig. 4 (a), where we can see strong localization along the open-star-polygonal orbit. The far-field pattern of this mode is shown in Figs. 4 (c) and 4 (d) (black curves). Figure 4 (c) shows the emission to the left side of the cavity (the side of $V_{0}^{\prime}$ ), while Fig. 4 (d) shows that to the right side (the side of $V_{11}^{\prime}$ ). We can confirm that there is a bi-directional emission that is in good agreement with the directions of the two attached linear waveguides (i.e., $\theta=0$ degrees in Fig. 4 (c), while $\theta=27.7$ degrees in Fig. 4 (d)). Note that the far-field patterns are normalized.

Unexpectedly, we found another type of high quality factor mode in the resonance distribution (labeled (b) in Fig. 3). Its wave function is shown in Fig. 4 (b), and its far-field patterns are shown in Figs. 4 (c) and 4 (d) (gray curves). A comparison between polygonal periodic orbits and the wave function pattern did not reveal a single dominant periodic orbit that explains the wave function pattern. However, the wave function appears to be localized along straight ray segments, especially those of unstable triangular (three-bounce) orbits. Such a mode might be understood as the scar mode [18]. This localization is interesting in itself, and deserves further investigation, but this will constitute future work, since our main focus here is on the star modes.

Gaussian optical theory [17] states that the beam waist spot size and position are directly related to the stability of the periodic orbit. For the open-star-polygonal orbit, the waveguide ends $V_{0}^{\prime}$ and $V_{11}^{\prime}$ are the beam waist positions, and their spot sizes are estimated as

$$
w=\tilde{w} \frac{r_{0}}{\sqrt{k r_{0}}},
$$

where $\tilde{w}=0.961$ for the beam waist at $V_{0}^{\prime}$, while $\tilde{w}=$ 0.898 for the beam waist at $V_{11}^{\prime}$. The beam divergence $\theta_{d}$ in the far field can be estimated by the formula $\theta_{d} \approx$ $\lambda /(\pi w)=2 /\left(\tilde{w} \sqrt{k r_{0}}\right)$, where $w$ is the beam spot size. This formula yields $\theta_{d}=8.0$ degrees for the emission at $V_{0}^{\prime}$, and $\theta_{d}=8.5$ degrees for the emission at $V_{11}^{\prime}$. On the other hand, for the far-field data obtained with the BEM calculation, we found that $\theta_{d}=11.8$ degrees for the emission at $V_{0}^{\prime}$ [black curve in Fig. 4(c)], while $\theta_{d}=$ 14.7 degrees for the emission at $V_{11}^{\prime}$ [black curve in Fig. 4(d)]. We consider that this broadening of the peaks is mainly caused by the deformation of the Gaussian beams due to the finiteness of the wavelength and the existence of ray chaos.

For the 10 star modes that we detected in our BEM calculations, we found an almost regular modal spacing that fluctuated from 0.0492 to 0.0540 . We consider this relatively large fluctuation to be related to dynamical tunneling [19], which is known generally to occur in ray- chaotic microcavities $20-22$, resulting in some portion of the intensity being leaked to ray chaotic orbits and spreading throughout the cavity. Nevertheless, we found that the average modal spacing is $\Delta k r_{0}=0.0511$, which closely agrees with a theoretical estimate based on the optical path length, i.e., $\Delta k r_{0}=\pi /\left(n L_{*}\right)=0.0512$, where $L_{*}=18.596$ is the (one-way) path length of the openstar-polygonal orbit normalized by $r_{0}$.

As for the decay rates, the average value of $\operatorname{Im} k r_{0}$ for the 10 star modes is -0.0120 . In the short-wavelength limit, $\operatorname{Im} k r_{0}$ can be approximately expressed as [17]

$$
\operatorname{Im} k r_{0}=\frac{\ln (1 / \mathcal{R})}{2 n L_{*}},
$$

where $\mathcal{R}=0.286$ is the Fresnel reflection coefficient for normal incidence and TE-polarization. This formula yields $\operatorname{Im} k r_{0}=-0.0102$. The average decay rate of the actual star modes is about $20 \%$ larger than this theoretical estimate. This effect can also be considered to result from the dynamical tunneling. That is, there is an additional channel for the leakage, or an emission that is formed by the dynamical tunneling [20].

\section{EXPERIMENTAL DATA}

We fabricated GaAs single-quantum-well (SQW) microcavity lasers whose cavity shape is defined in Fig. 1 (a) with average diameters of $r_{0}=30 \mu \mathrm{m}$ and $r_{0}=50 \mu \mathrm{m}$. The GaAs SQW heterostructures were grown by metalorganic chemical-vapor deposition (MOCVD) on an ntype GaAs substrate with a $1.5-\mu \mathrm{m} n-\mathrm{Al}_{0.5} \mathrm{Ga}_{0.5}$ As lower cladding layer, a $0.2-\mu \mathrm{m} n-\mathrm{Al}_{x} \mathrm{Ga}_{1-x} \mathrm{As}(x=0.5-0.2)$ graded region, a 10-nm GaAs quantum well layer, a 0.2$\mu \mathrm{m} p-\mathrm{Al}_{x} \mathrm{Ga}_{1-x} \mathrm{As}(x=0.2-0.5)$ graded region, a $1.5-\mu \mathrm{m}$ $p$ - $\mathrm{Al}_{0.5} \mathrm{Ga}_{0.5} \mathrm{As}$ upper cladding layer, a $0.2-\mu \mathrm{m} p$-GaAs contact layer, and a 400-nm $\mathrm{SiO}_{2}$ layer. The etching depth from the top of the contact layer was about 4.2 $\mu \mathrm{m}$, which was $2.3 \mu \mathrm{m}$ below the active layer. The contact area was etched through the $\mathrm{SiO}_{2}$ layer, and the $p$-electrode metal was formed over the contact area and part of the surrounding $\mathrm{SiO}_{2}$ layer using a liftoff process (see Ref. 23] for details of the fabrication process). Figure 1(c) shows the contact area shape, which was designed to fit to the star modes. It mainly consists of an annular area, whose outer and inner boundaries have the shape defined by Eq. (1) with the average radii $r_{0}-5 \mu \mathrm{m}$ and $r_{0} / 2$, respectively, where $r_{0}=30 \mu \mathrm{m}$ or $50 \mu \mathrm{m}$. In addition, along the two linear waveguides, $2 \mu \mathrm{m}$-width linear contact areas are formed. The $5 \mu \mathrm{m}$ margin for the outer boundary of the contact area was necessary for the liftoff process used for forming the $p$-electrode metal 23]. The lasers were electrically pumped by current injection with continuous wave $(\mathrm{CW})$ pumping at room temperature. The lasing threshold was $30 \mathrm{~mA}$ for the device with $r_{0}=30 \mu \mathrm{m}$, and $37 \mathrm{~mA}$ for the device with $r_{0}=50 \mu \mathrm{m}$. This implies that the threshold current density is $2.4 \mathrm{kA} / \mathrm{cm}^{2}$ for $r_{0}=30 \mu \mathrm{m}$, and $0.84 \mathrm{kA} / \mathrm{cm}^{2}$ for 

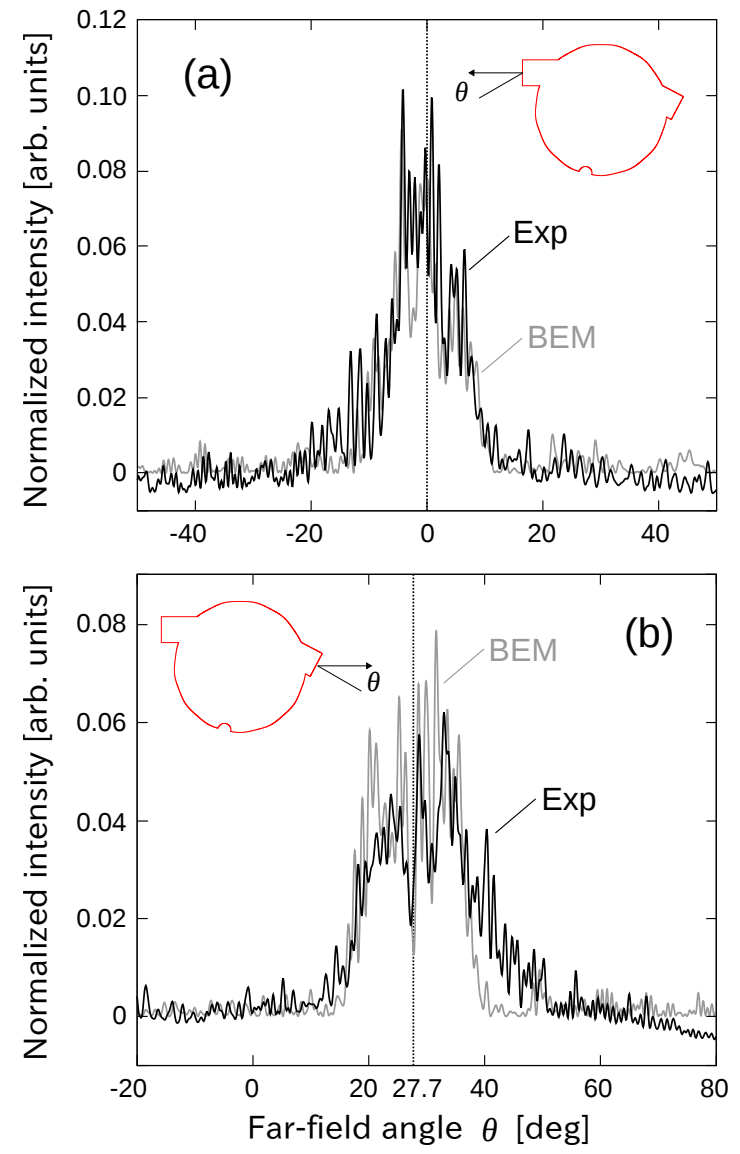

FIG. 5: (Color online) Far-field patterns (normalized) for a device with $r_{0}=30 \mu \mathrm{m}$ : (a) emission to the left side of the cavity, (b) emission to the right side. The direction of the waveguide is $\theta=0$ degrees in (a), while it is $\theta=27.7$ degrees in (b). Experimental data for a pumping current of $90 \mathrm{~mA}$ are plotted with black curves, while numerical data obtained with the BEM calculation are plotted with gray curves.

$r_{0}=50 \mu \mathrm{m}$ (the contact area is approximately given by $\left.\pi\left[\left(r_{0}-5\right)^{2}-r_{0}^{2} / 4\right]\right)$. We consider that the higher threshold current density for $r_{0}=30 \mu \mathrm{m}$ is due to the threshold increase by a temperature rise under $\mathrm{CW}$ pumping, which is expected to be more significant for a smaller cavity.

In our experiments, we observed bi-directional emissions in good agreement with the directions of the two attached waveguides. Figure 5 (black curves) shows measured far-field patterns for the device with $r_{0}=30 \mu \mathrm{m}$ when the pumping current was $90 \mathrm{~mA}$. The far-field patterns were measured by rotating the photodetector with a slit around the device. The slit width was $0.5 \mathrm{~mm}$ and the radius of the rotation was $30 \mathrm{~cm}$, which yielded a resolution of 0.1 degrees. As the result of the Lloyd's mirror effect, the direct output from the active layer and the output once reflected at the GaAs substrate produced an interference pattern on the plane vertical to the horizontal plane where the device was placed. The height of the photodetector was adjusted to the position of the first-order interference to maximize the detected inten-
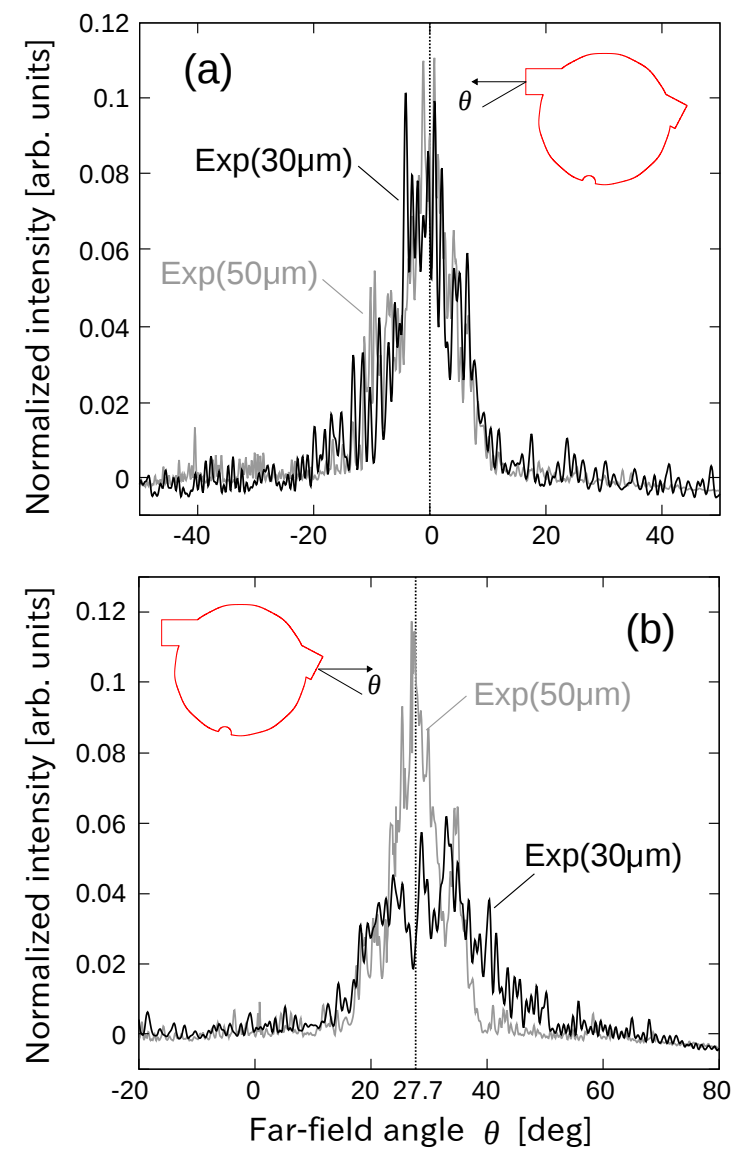

FIG. 6: (Color online) Far-field patterns (normalized) for devices with $r_{0}=30 \mu \mathrm{m}$ and $r_{0}=50 \mu \mathrm{m}$ : (a) emission to the left side of the cavity, (b) emission to the right side. The direction of the waveguide is $\theta=0$ degrees in (a), while it is $\theta=27.7$ degrees in (b). Experimental data for $r_{0}=30 \mu \mathrm{m}$ are plotted with black curves, while those for $r_{0}=50 \mu \mathrm{m}$ are plotted with gray curves. The pumping current is $90 \mathrm{~mA}$ in all cases.

sity.

The uniform background radiation caused by spontaneous emission is subtracted from the experimental data in Fig. 5, and the far-field patterns are normalized. When we compare these results with the numerical results of the BEM calculation superposed in Fig. 5 (gray curves), we can confirm good agreement between the experimental and theoretical data. Note that the condition for the BEM calculation was the same as that for the experiment (i.e., $r_{0}=30 \mu \mathrm{m}$ ). Figure 6 shows measured far-field patterns for the device with $r_{0}=50 \mu \mathrm{m}$ (gray curves) together with the results for the device with $r_{0}$ $=30 \mu \mathrm{m}$ (black curves). We again see good agreement with the theoretical prediction, as well as a slight far-field peak narrowing for $r_{0}=50 \mu \mathrm{m}$, especially in Fig. 6 (b). This is consistent with the fact that the beam divergence $\theta_{d}$ is inversely proportional to $\sqrt{k r_{0}}$.

Figure 7 (a) shows the optical spectrum obtained for the device with $r_{0}=30 \mu \mathrm{m}$ when pumped below the 

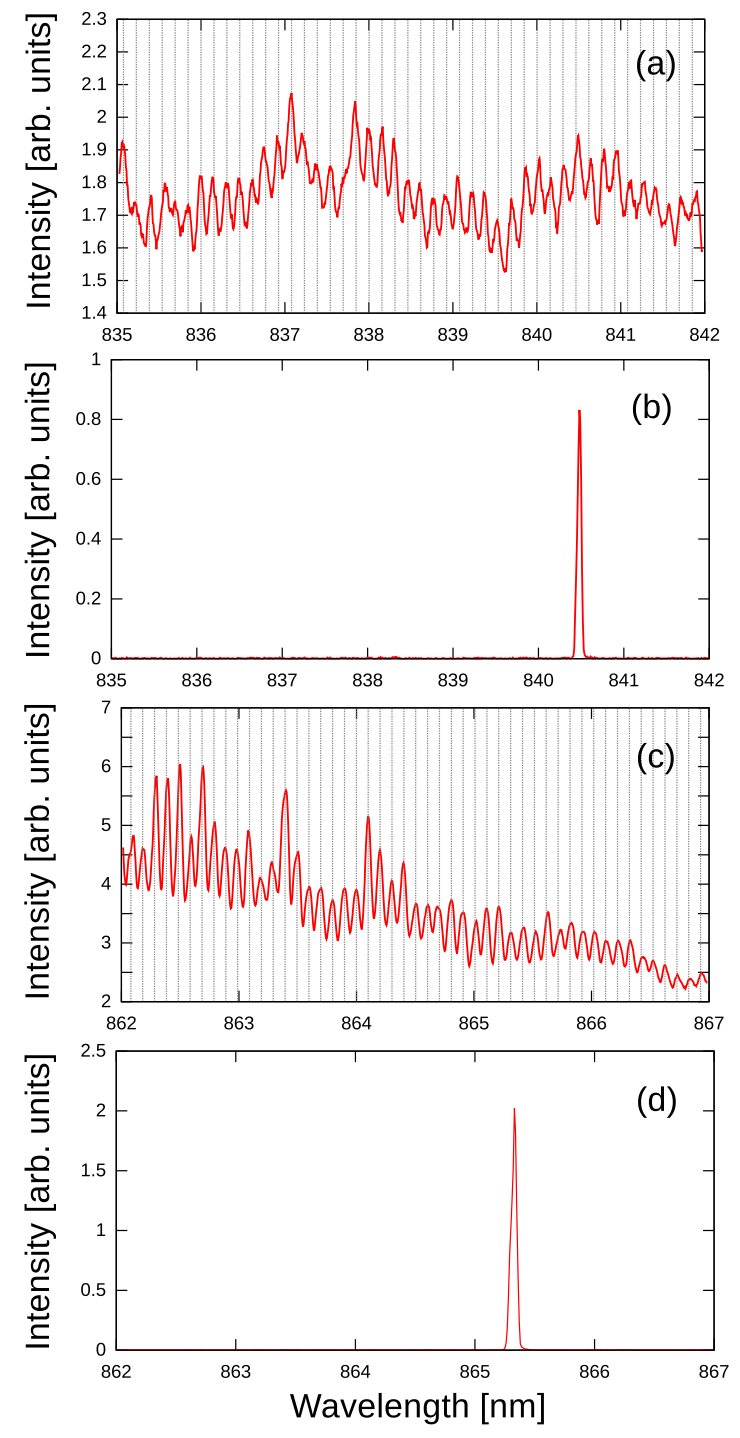

FIG. 7: (Color online) Optical spectra: (a) $r_{0}=30 \mu \mathrm{m}$ and below the threshold, (b) $r_{0}=30 \mu \mathrm{m}$ at $90 \mathrm{~mA}$, (c) $r_{0}=50 \mu \mathrm{m}$ and below the threshold (d) $r_{0}=50 \mu \mathrm{m}$ at $90 \mathrm{~mA}$. In (a) and (c), respectively, equidistant vertical dotted lines with 0.154 $\mathrm{nm}$ and $0.101 \mathrm{~nm}$ spacings are plotted as an eye guide.

threshold. In the spectral measurement, the photodetector was placed in the direction of the left linear waveguide. We interpret the peaks we observed here as the cavity modes coupled with the amplified spontaneous emission. We can see a regular modal spacing $\Delta \lambda=0.154 \mathrm{~nm}$. This value is in excellent agreement with the theoretical estimate of $0.153 \mathrm{~nm}$, which takes account of the dispersion, i.e., $\Delta \lambda=\lambda^{2} /\left[2 n L_{*} r_{0}(1-(\lambda / n)(d n / d \lambda)]\right.$, where $\lambda$ $=0.84 \mu \mathrm{m}$ is the lasing wavelength, $L_{*} r_{0}=557.89 \mu \mathrm{m}$ is the (one-way) path length of the open-star-polygonal orbit, and $d n / d \lambda=-1.0 \mu \mathrm{m}^{-1}$ is the dispersion [24]. Figure 7 (c) shows an optical spectrum for the device with $r_{0}=50 \mu \mathrm{m}$ when pumped below the threshold. The measured modal spacing is $\Delta \lambda=0.101 \mathrm{~nm}$, while the theoretical estimate based on the path length is $\Delta \lambda=0.097 \mathrm{~nm}$.
From the above far-field and spectral data, we concluded that the star modes existed and were successfully excited in our fabricated devices. As for the unexpected high quality factor modes that we observed in the BEM calculation (i.e., the modes exemplified in Fig. (4 (b)), their existence cannot be confirmed in the spectra shown in Figs. 7 (a) and 7 (c). This might be due to our contact pattern [Fig. 1 (c)], which we designed to preferentially excite the star modes. Also, as can be seen in the spatial intensity pattern of the unexpected high quality factor mode shown in Fig. 4(b), it does not couple significantly with the linear waveguides. Thus, the photo detector placed in the direction of the waveguide might not be able to capture the emission from an unexpected high quality factor mode, even if it is excited.

Interestingly, when the devices are pumped above the threshold, we observed single-mode lasing, as shown in Fig. 7 (b) for the device with $r_{0}=30 \mu \mathrm{m}$ and in Fig. 7 (d) for the device with $r_{0}=50 \mu \mathrm{m}$, where the pumping currents were $90 \mathrm{~mA}$ in both cases. For chaotic cavity lasers, single-mode lasing has been experimentally observed with CW pumping 25 28]. For chaotic cavity lasers, it has been numerically observed that nonlinear interactions among modes reduce the number of lasing modes [29], and experimentally observed single-mode lasing is attributed to large modal overlaps between resonant modes [28].

The excitation of multiple longitudinal Gaussian beam modes has been observed with CW pumping for a stable Fabry-Perot cavity laser [30]. This is in contrast to our results. We consider this difference to be caused by the fact that our Gaussian beam modes are affected by dynamical tunneling. As discussed in Sec. III the intensity pattern of our Gaussian beam mode was slightly deformed from an exact Gaussian beam because of the intensity leakage throughout the cavity. This can result in a fluctuation in the decay rates (i.e., $\operatorname{Im} k r_{0}$ ) as well as an increase in the modal overlaps. The former makes a certain mode preferential for lasing, while the latter can cause the suppression of the other lasing modes. We expect these effects to lead to single-mode lasing, although more thorough investigations are needed for a definitive understanding.

\section{SUMMARY AND DISCUSSION}

We studied both theoretically and experimentally a deformed microdisk designed for long path formation. We performed resonant mode analysis to reveal that the long-path modes were characterized by high quality factors. For fabricated deformed microdisks with average radii $r_{0}=30 \mu \mathrm{m}$ and $50 \mu \mathrm{m}$, we presented measured farfield and spectral data that confirmed the path length of $18.596 \times r_{0}$.

Based on this approach, the path length can be in principle extended by increasing the size parameter $r_{0}$ or the number of vertices $Q$, although the finite wavelength lim- 


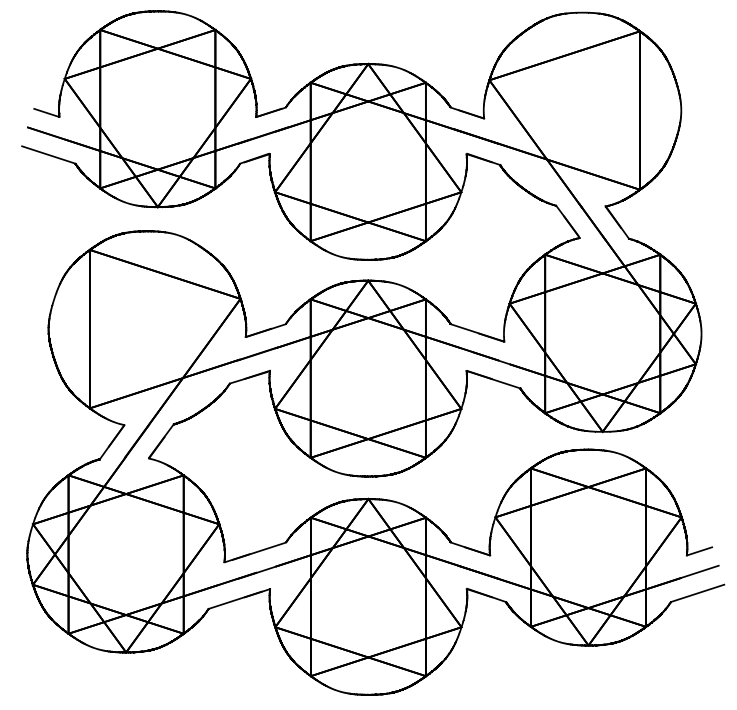

FIG. 8: Long path formation by coupling 9 identical deformed disks with $Q=10$ and an average radius $r_{0}$. The total path length is about $107 \times r_{0}$, whereas the entire system length is about $6 \times r_{0}$.

its the maximum $Q$ value, since each vertex needs to be resolved by the light wave so that a path is distinctly formed. Another interesting way of extending a path is to couple many cavities as illustrated in Fig. 8, where 9 identical deformed disks with $Q=10$ and an average radius $r_{0}$ are connected. In this example, the total path length adds up to about $107 \times r_{0}$, whereas the entire system length is about $6 \times r_{0}$. We performed a linear stability analysis as described in Sec. III for this self-retracing periodic orbit, and confirmed the existence of cavity parameter regimes where the periodic orbit becomes stable. In this way, we can use two dimensions efficiently to obtain a long path. Conventionally, a spiral geometry has been adopted for long path formation [31]. For a spiral waveguide, bending losses are inevitable, limiting the maximum curvature of a spiral and thus the entire system size. Our approach may be useful as an alternative that is free from bending losses.

The cavity studied here can also be viewed as the one exhibiting relatively good emission directionality. Bidirectionality is inevitable for our cavity design based on the open-star-polygonal periodic orbit. However, we believe that unidirectionality can be achieved, by letting one of the waveguide facets coincide with a cleavage facet, and coating it with high reflectivity film.
[1] J. U. Nöckel and A. D. Stone, Nature 385,45 (1997).

[2] C. Gmachl, F. Capasso, E. E. Narimanov, J. U. Nöckel, A. D. Stone, J. Faist, D. L. Sivco, and A. Y. Cho, Science 280, 1556 (1998).

[3] N. Chernov and R. Markarian, Chaotic Billiards (Mathematical Surveys and Monographs, Vol. 127) (AMS, 2006).

[4] H. G. L. Schwefel, H. E. Tureci, A. D. Stone, and R. K. Chang, in Optical Microcavities, edited by K. Vahala (World Scientific, Singapore, 2004).

[5] T. Harayama and S. Shinohara, Laser Photonics Rev. 5, 247 (2011).

[6] H. Cao and J. Wiersig, Rev. Mod. Phys. 87, 61 (2015).

[7] E. Narimanov, J. Fan, and C. Gmachl, in Technical Digest of Quantum Electronics and Laser Science Conference, 1, 421 (2005).

[8] D. Qu and Z. Liu, and C. Gmachl, Appl. Phys. Lett., 93, 014101 (2008).

[9] D. Qu and C. Gmachl, Phys. Rev. A 78, 033824 (2008).

[10] J. U. White, J. Opt. Soc. Am. 32, 285 (1942).

[11] D. R. Herriott and H. J. Schulte, Appl. Opt. 4, 883 (1965).

[12] S. Shinohara, S. Sunada, T. Fukushima, T. Harayama, K. Arai, and K. Yoshimura, Appl. Phys. Lett. 105, 151111 (2014).

[13] A. Uchida, Optical Communication with Chaotic Lasers (Wiley-VCH, Weinheim, 2012).

[14] J. Ohtsubo, Semiconductor Lasers (Springer-Verlag, Berlin, 2008).

[15] S. Sunada, T. Fukushima, S. Shinohara, T. Harayama, K. Arai, and M. Adachi, Appl. Phys. Lett. 104, 241105 (2014).

[16] J. Wiersig, J. Opt. A: Pure Appl. Opt. 5, 53 (2003).

[17] H. E. Tureci, H. G. L. Schwefel, A. D. Stone, and E. E.
Narimanov, Opt. Express 10, 752 (2002).

[18] E. J. Heller, Phys. Rev. Lett. 53, 1515 (1984).

[19] M. J. Davis and E. J. Heller, J. Chem. Phys. 75, 246 (1981).

[20] S. Shinohara, T. Harayama, T. Fukushima, M. Hentschel, T. Sasaki, and E. E. Narimanov, Phys. Rev. Lett. 104, 163902 (2010).

[21] J. Yang, S. -B. Lee, S. Moon, S. -Y. Lee, S. W. Kim, T. T. Anh Dao, J. -H. Lee, and K. An, Phys. Rev. Lett. 104, 243601 (2010).

[22] Q. Song, L. Ge, B. Redding, and H. Cao, Phys. Rev. Lett. 108, 243902 (2012).

[23] T. Fukushima and T. Harayama, IEEE J. Sel. Top. Quantum Electron. 10, 1039 (2004).

[24] H. C. Casey Jr. and M. B. Panish, Heterostructure Lasers (Academic Press, New York, 1978).

[25] R. Audet, M. A. Belkin, J. A. Fan, B. G. Lee, K. Lin, F. Capasso, E. E. Narimanov, D. Bour, S. Corzine, J. Zhu, and G. Höfler, Appl. Phys. Lett. 91, 131106 (2007).

[26] M. -W. Kim, C. -H. Yi, S. Rim, C. -M. Kim, J. -H. Kim, and K. -R. Oh, Opt. Express 20, 13651 (2012).

[27] S. Sunada, T. Fukushima, S. Shinohara, T. Harayama, and M. Adachi, Phys. Rev. A 88, 013802 (2013).

[28] S. Sunada, S. Shinohara, T. Fukushima, and T. Harayama, Phys. Rev. Lett. 116, 203903 (2016).

[29] S. Sunada, T. Harayama, and K. S. Ikeda, Phys. Rev. E 71, 046209 (2005).

[30] T. Fukushima, S. Sunada, T. Harayama, K. Sakaguchi, and Y. Tokuda, Appl. Opt. 51, 2515 (2012).

[31] H. Lee, T. Chen, J. Li, O. Painter, and K. J. Vahala, Nat. Commun. 3, 867 (2012). 